\title{
Les mauvais traitements psychologiques subis par les adolescents et adolescentes . . . des mots qui frappent!
}

\author{
Cynthia Bêty \\ Centre hospitalier universitaire de Québec \\ Francine Lavoie \\ Université Laval \\ Danielle Nadeau \\ Centre Jeunesse de Québec-Institut universitaire
}

\begin{abstract}
RÉSUMÉ
La recherche se penche sur les perceptions des mauvais traitements psychologiques (MTP) chez une population en ayant été victime et recevant les services d'un Centre Jeunesse. L'étude a eu pour objectifs (a) d'investiguer l'attribution que les jeunes font des causes des MTP, leur perception de contrôle et leur perception actuelle de l'abuseur, et (b) d'évaluer si les perceptions diffèrent en fonction du type de lien entre la victime et l'abuseur. Trente-six incidents critiques ont été recueillis auprès des 19 participants et participantes et ont été soumis à une analyse de contenu. Cette étude met en lumière la souffrance des victimes de MTP et montre que la violence intrafamiliale et la violence extrafamiliale sont perçues de façon différente par les victimes.
\end{abstract}

Mots clés : mauvais traitements psychologiques, perceptions, adolescents, Centre Jeunesse

Cynthia Bêty, PhD, Psychologue, Centre hospitalier universitaire de Québec, Québec. Francine Lavoie, PhD, Professeure titulaire, École de psychologie, Université Laval, Québec. Danielle Nadeau, PhD, Psychologue-chercheur, Centre Jeunesse de QuébecInstitut universitaire, Québec.

Les auteures désirent remercier Marie-Hélène Gagné et Louise Bujold pour leurs recommandations quant à cette étude. Nous remercions également Jalila Jbilou pour l'aide apportée lors de l'analyse qualitative. Les auteures tiennent aussi à remercier le Centre jeunesse et les intervenants et intervenantes pour leur soutien à la recherche et le groupe ÉVISSA, subventionné par le Fonds québécois de la recherche sur la société et la culture (FQRSC).

La correspondance concernant cet article devrait être acheminée à Cynthia Bêty, PhD, École de psychologie, 2325 rue des Bibliothèques, Université Laval, Québec (Québec) G1V 0A6. Tél : 418-570-4610. Courriel : cynthia_bety@hotmail.com 


\begin{abstract}
This qualitative study investigated perceptions of Psychological Maltreatment (PM) with 19 adolescent victims referred to a child welfare agency. The research objectives were: (a) to explore youths' causal attributions of PM, their perceived control of the PM situation, and their perceptions of the abuser; and (b) to document whether these perceptions differ as a function of the nature of the relationship between the youth and his or her abuser. Thirty-six critical incidents were investigated through semi-structured interviews with 19 participants. Interview transcripts were subjected to content analysis. Results highlight the subjective suffering of PM victims in this population and the difference in perceptions of this kind of violence according to whether intrafamilial or extrafamilial PM is involved.
\end{abstract}

Keywords: Psychological Maltreatment, perceptions, adolescents, child welfare agency

L'Organisation mondiale de la santé mentionne que les mauvais traitements psychologiques (MTP) sont moins abordés que la violence sexuelle et physique dans la littérature scientifique (Krug, Dahlberg, Mercy, Zwi et Lozano-Ascencio, 2002). En fait, les MTP constituent la dernière forme de violence à laquelle les chercheurs et les autorités se sont attardés (Brassard et Donovan, 2006). Ils « se traduisent notamment par de l'indifférence, du dénigrement, du rejet affectif, de l'isolement, des menaces, de l'exploitation [. . .] ou par l'exposition à la violence conjugale ou familiale » (Québec, 2007, art. 38c). On sait aujourd'hui que les MTP touchent une proportion importante d'enfants et d'adolescents et adolescentes (e.g., Clément, Chamberland, Côté, Dubeau et Beauvais, 2005), particulièrement ceux et celles recevant les services de la Protection de la Jeunesse (Tourigny et al., 2002), ce qui nous motive à étudier cette population de plus près. D'autre part, même s'il est connu que cette forme de violence est associée à plusieurs conséquences au plan de la santé mentale et des comportements (e.g., Gagné, Drapeau, Melançon, Saint-Jacques et Lépine, 2007; Sneddon, 2003; Webb, Heisler, Call, Chickering et Colburn, 2007), nous en savons encore peu sur le vécu subjectif des victimes dans l'état actuel des connaissances.

Trois études qualitatives font toutefois exception. Au Québec, des auteures ont réalisé une analyse de contenu de demandes faites par des adolescents et adolescentes de la population générale à un service d'aide concernant la violence psychologique parentale afin de mieux comprendre comment cette violence est vécue (Gagné, Melançon, Pouliot-Lapointe, Lavoie et Roy, 2010). DeRobertis (2004) s'est quant à lui penché sur le point de vue des victimes de MTP chroniques et causés par la figure maternelle. Pour ce faire, cinq adultes ont été invités à décrire les MTP subis dans l'enfance et leurs impacts. Concernant l'exposition uniquement à la violence conjugale, qui constitue une forme de MTP, Joseph, Govender et Bhagwanjee (2006) avaient pour objectif de comprendre comment les enfants s'adaptent à la violence conjugale à laquelle ils sont exposés. Afin de répondre à leur question de recherche, les auteurs ont adopté une approche phénoménologique et ont interrogé cinq enfants âgés entre 8 et 12 ans. Dans l'ensemble, ces trois études ont l'avantage de montrer que les MTP entrainent une souffrance importante et que les perceptions des MTP sont très variées. En plus, elles nous donnent accès à la richesse du vécu subjectif des victimes, ce qui est peu présent du côté des études quantitatives. Connaître le vécu subjectif des victimes est essentiel. D'ailleurs, selon une récente prise de position de l'American Psychological Association, le point de vue des personnes touchées par un problème doit être davantage pris en compte (Anton, 2010). Investiguer l'évaluation subjective d'un abus telle que révélée par la victime s'avère primordial d'autant plus que cette variable est importante selon les cliniciens et cliniciennes (e.g., Beck, 1995; Cohen et Mannarino, 2002; Feiring, 2005). Comme l'indique Fortin (2005), 
analyser le point de vue des victimes de violence nous permet de comprendre davantage leurs réactions et leurs difficultés d'adaptation.

Les trois études qualitatives mentionnées plus tôt sont limitées à la violence parentale et, dans un cas, les données sont tirées de brefs textes (Gagné et al., 2010). Notre étude recourt en outre aux entrevues permettant une analyse plus approfondie que l'étude des transcriptions de demandes d'aide de Gagné et al. (2010). Les perceptions liées aux MTP subis sont ici investiguées chez une population à risque et susceptible d'avoir subi des MTP, soit des jeunes recevant les services d'un Centre Jeunesse ${ }^{1}$ (CJ) et ayant fait une tentative de suicide récente. Non seulement les écrits montrent un lien entre les MTP et les comportements suicidaires (e.g., Bifulco, Moran, Baines, Bunn et Stanford, 2002; Klomek, Marroco, Kleinman, Schonfeld et Gould, 2007; Lyon et al., 2000; Nilsen et Conner, 2002), cette clientèle, souvent négligée, cumule bien des problèmes et exprime sa détresse de diverses façons tout en tentant de s'adapter (Bêty, Lavoie et Nadeau, 2011). En plus, elle constitue une priorité en recherche au CJ et mérite donc que l'on s'y attarde de plus près. Leur donner la parole est un objectif important de notre recherche. Les victimes ne sont pas ici interrogées sur leur tentative de suicide, mais sur les MTP qu'ils ont subis. C'est dans une étude plus large que le lien entre les MTP et la tentative de suicide fut investigué auprès des participants et participantes (Bêty, 2012).

Parmi les variables subjectives existantes, nous nous préoccupons de l'attribution des causes, de la perception de contrôle et des perceptions actuelles face à l'abuseur (homme ou femme). Ces variables ont été sélectionnées suite à une recension d'écrits portant sur les perceptions de la violence subie en général et la présence de différents symptômes de détresse psychologique. La littérature montre que diverses difficultés au plan de la santé mentale sont, entre autres, reliées au fait d'attribuer des événements négatifs à des causes internes, stables et globales (e.g., Feiring et Cleland, 2007; Lo, Ho et Hollon, 2010; Mezulis, Funasaki et Hyde, 2011), et à la perception de ne pas avoir le contrôle (Gençoz, Vatan, Walker et Lester, 2008; Lester et Walker, 2007). Concernant les perceptions actuelles face à l'abuseur, les populations investiguées, les perceptions évaluées et le type d'abus subi par les participants et participantes varient d'étude en étude, ce qui rend difficile l'exercice de comparer les conclusions. Ces aspects gagnent à être documentés afin de mieux comprendre comment les victimes de MTP perçoivent l'abuseur et, ainsi, améliorer les interventions offertes.

\section{Objectifs}

La présente recherche qualitative se penche sur les perceptions des MTP chez une population clinique juvénile qui en fut victime. Plus précisément, elle a pour objectifs (a) d'investiguer l'attribution que les jeunes font des causes des MTP, leur perception de contrôle, leur perception actuelle de l'abuseur, et (b) d'évaluer si les perceptions diffèrent en fonction du type de lien entre la victime et l'abuseur.

\section{MÉTHODOLOGIE}

\section{Participants et participantes}

L'échantillon est composé de 19 adolescents et adolescentes, soit 13 garçons et 6 filles, âgés entre 12 et 18 ans $(M=15,74, E ́ T=1,33)$. Les participants et participantes recevaient les services d'un CJ au moment de l'entrevue et avaient tous fait une tentative de suicide au cours des 3 années précédant la rencontre. Les données démographiques se retrouvent dans le tableau 1. 
Tableau 1

Description de l'échantillon réparti selon le sexe

\begin{tabular}{|c|c|c|c|c|}
\hline \multirow[t]{3}{*}{ Variable } & \multicolumn{4}{|c|}{ Sexe } \\
\hline & \multicolumn{2}{|c|}{ Filles } & \multicolumn{2}{|c|}{ Garçons } \\
\hline & $n$ & $\%$ & $n$ & $\%$ \\
\hline \multicolumn{5}{|l|}{ Âge } \\
\hline 12 à 13 ans & 0 & 0,0 & 1 & 7,7 \\
\hline 14 à 15 ans & 2 & 33,3 & 4 & 30,8 \\
\hline 16 à 18 ans & 4 & 66,7 & 8 & 61,5 \\
\hline \multicolumn{5}{|l|}{ Scolarité* $^{*}$} \\
\hline Secondaire 1 & 1 & 16,7 & 5 & 38,5 \\
\hline Secondaire 2 & 3 & 50,0 & 4 & 30,8 \\
\hline Secondaire 3 & 0 & 0,0 & 1 & 7,7 \\
\hline Secondaire 4 & 1 & 16,7 & 2 & 15,4 \\
\hline Secondaire 5 & 0 & 0,0 & 1 & 7,7 \\
\hline \multicolumn{5}{|l|}{ Formation préparatoire } \\
\hline au marché du travail & 1 & 16,7 & 0 & 0,0 \\
\hline \multicolumn{5}{|l|}{ Milieu de vie } \\
\hline Centre de réadaptation & 3 & 50,0 & 12 & 92,3 \\
\hline Famille d'origine & 1 & 16,7 & 1 & 7,7 \\
\hline Foyer de groupe & 1 & 16,7 & 0 & 0,0 \\
\hline Autre & 1 & 16,7 & 0 & 0,0 \\
\hline \multicolumn{5}{|l|}{ Origine } \\
\hline Québécoise & 4 & 66,7 & 12 & 92,3 \\
\hline Autre & 2 & 33,3 & 1 & 7,7 \\
\hline \multicolumn{5}{|l|}{ Composition de la famille } \\
\hline Reconstituée & 6 & 100,0 & 10 & 76,9 \\
\hline Biparentale intacte & 0 & 0,0 & 1 & 7,7 \\
\hline Famille adoptive & 0 & 0,0 & 1 & 7,7 \\
\hline Famille séparée & 0 & 0,0 & 1 & 7,7 \\
\hline
\end{tabular}

* Le niveau de scolarité le plus élevé a été retenu. 
Les participantes et participants rencontrés constituent une population à risque. Parmi les milieux de vie décrits au tableau 1, on note que 15 d'entre eux étaient hébergés en centre de réadaptation ${ }^{2}$ lors de la rencontre, ce qui témoigne de leurs difficultés comportementales, psychosociales et/ou familiales. Ce sont des adolescents et adolescentes qui avaient vécu de nombreux événements stressants au cours de l'année précédant la rencontre. En effet, selon ce que les jeunes ont mentionné en cours d'entrevue, la fugue, la tentative de suicide, les démêlés avec la justice, le fait d'être suspendu de l'école, les problèmes d'argent éprouvés par la famille, les placements et le fait de s'être battu figurent parmi les épisodes marquants. Aussi, deux des adolescents étaient placés sous la Loi sur le système de justice pénale pour adolescents, c.-à-d., ils faisaient face à des accusations au pénal.

\section{Procédure de recrutement}

Le recrutement a eu lieu en 2008 au sein d'un Centre Jeunesse desservant une clientèle urbaine et rurale de 680000 habitants (Institut de la statistique du Québec, 2010). Les intervenants et les intervenantes de ce Centre Jeunesse étaient invités à référer des jeunes âgés de 12 ans et plus et ayant tenté de se suicider au cours des 3 années précédant la rencontre. La tentative suicidaire est définie comme :

tout acte délibéré visant à accomplir sur sa propre personne un geste de violence dont l'issue n'est pas fatale. Ainsi, différents gestes sont décrits : phlébotomie, intoxication au gaz, ingestion d'une substance toxique ou de médicaments ... Cet acte doit être inhabituel : les conduites addictives (alcool, drogues ....) sont donc exclues de cette définition ainsi que les automutilations répétées et les refus de s'alimenter. (Organisation mondiale de la santé, 2004, p. 105)

Les jeunes perçus par leur intervenant ou intervenante comme étant trop perturbés pour participer étaient exclus de l'étude. Le recrutement a pris fin lorsque 19 adolescents et adolescentes avaient été recrutés puisque la saturation des catégories avait alors été atteinte.

Par ailleurs, ce projet de recherche a été approuvé par le Comité d'éthique de recherche du Centre Jeunesse où s'est déroulé le recrutement et c'est la chercheure principale qui était responsable de l'obtention des consentements écrits d'un(e) des parents et du jeune. Aussi, bien que demander aux jeunes de nous raconter des épisodes où ils ont été victimisés soulève des enjeux éthiques et légaux (Hurley et Underwood, 2002; Putnam, Liss et Landsverk, 1996), il semble important de préciser que l'ensemble des participantes et participants rencontrés se sentaient bien à la fin de l'entrevue et mentionnaient même qu'ils étaient contents d'avoir pu en parler.

\section{Guide d'entrevue}

Parmi les méthodes existantes, c'est l'entrevue semi-dirigée qui fut sélectionnée puisqu'elle permettait d'avoir accès à un matériel riche en informations ainsi qu'aux expériences tant cognitives qu'émotionnelles des participants et participantes (Frechtling, 2002). La structure de l'entrevue s'inspirait de la technique de l'incident critique, proposée à l'origine par Flanagan (1954). Les jeunes ont été invités à donner deux exemples de MTP : le premier ayant été subi au cours des 3 mois précédant la rencontre et le deuxième constituant l'épisode le plus stressant à vie. Ces deux exemples étaient au cœur de l'entrevue et les questions concernant les perceptions y faisaient référence. 
Afin d'évaluer l'attribution des causes, tout comme Feiring, Taska et Chen (2002) l'ont fait auprès d'enfants victimes d'abus sexuels, il était demandé aux participants et participantes de nous expliquer pourquoi, selon eux, les MTP avaient eu lieu. En ce qui concerne la perception de contrôle définie comme « la croyance en ses propres capacités pour influencer les événements de vie personnels ou pour contrôler les résultats » (traduction libre, O’Neill et Kerig, 2000, p. 1037), nous avons demandé aux participants et participantes s'ils avaient eu l'impression de pouvoir changer la suite des événements ou la situation, ou encore, s'ils percevaient avoir eu le contrôle. Finalement, afin de documenter leur perception de l'abuseur, les jeunes ont été invités à nous décrire l'abuseur tel qu'ils le perçoivent présentement et à nous dire ce qu'ils ressentent actuellement à son égard. Les informations sur le type de lien entre la victime et l'abuseur découlent de l'analyse. Les catégories ne sont donc pas préétablies.

\section{Analyse de contenu}

Tel que le recommandent Miles et Huberman (2003), des fiches de synthèse d'entretiens (type de collaboration, etc.) ont été rédigées suite aux rencontres avec les participants et participantes et consultées avant l'analyse. Les verbatims ont été soumis à une analyse de contenu qui est « une méthode de classification ou de codification dans diverses catégories des éléments du document analysé pour en faire ressortir les différentes caractéristiques en vue d'en mieux comprendre le sens exact et précis » (L'Écuyer, 1990, p. 50). L'analyse était du type catégories mixtes proposé par L'Écuyer (1990) et Giles (2002), et s'inspirait de Deslauriers (1991).

Par la suite, à l'aide du logiciel N'Vivo 7, des matrices ont été créées afin d'analyser séparément comment chaque incident critique a été vécu, qu'il soit récent ou le plus stressant. Nous avons alors fait des regroupements en fonction du type de lien entre la victime et l'abuseur afin d'examiner si les MTP sont perçus différemment selon ces types de lien (Bardin, 1991).

Différentes stratégies ont été utilisées afin d'assurer la validité et la fiabilité de notre démarche. Entre autres, la saturation des catégories a été atteinte, car l'analyse des derniers verbatims était répétitive (Paillé et Mucchielli, 2003) et aucune nouvelle donnée ne venait contredire les catégories déjà en place (Laperrière, 1994). De plus, la recherche a été supervisée par deux expertes dans le but d'assurer la neutralité et l'honnêteté de la chercheure principale (e.g., Creswell, 2007; Mertens, 2005).

\section{Présentation}

L'analyse a porté sur 36 incidents critiques. En effet, deux jeunes ont raconté un seul exemple de MTP, soit le plus stressant à vie, alors que les autres participants et participantes ont été en mesure de donner deux incidents critiques (un récent et le plus stressant). Une première analyse a permis de cerner qu'il s'agissait de violence intrafamiliale (abandon, rejet affectif, rabaissement, exposition à la violence conjugale ou familiale) et de violence extrafamiliale, ce qui inclut la violence commise par les pairs (menaces, rabaissement, rejet affectif), la violence causée par les intervenants et intervenantes (indifférence affective, rabaissement) et la violence commise par d'autres adultes (menaces, manipulation, exploitation).

La violence intrafamiliale regroupe les épisodes de violence survenus au sein de la famille, c.-à-d., les épisodes où le ou les abuseur(s) sont des membres de la famille tels les parents, les grands-parents, la 
fratrie, le beau-père ou la belle-mère. La violence extrafamiliale commise pas les pairs est définie comme un épisode de violence causé par une personne semblable à la victime quant à sa fonction et à sa situation sociale. Dans ce cas, l'abuseur est un autre adolescent ou adolescente connu ou non de la victime. La violence extrafamiliale faite par les intervenants et intervenantes réfère aux récits où l'abuseur est une personne travaillant dans le réseau public. Il peut donc s'agir des professionnel(le)s de la santé, d'agent(e)s de la paix ou d'employé(e)s du Centre Jeunesse. Finalement, quelques incidents critiques recueillis concernent la violence extrafamiliale causée par un autre adulte. Nous reviendrons sur l'analyse de ces quatre types de lien entre la victime et l'abuseur plus loin.

Les jeunes ont réagi de façon différente à l'un ou l'autre des deux exemples de MTP rapportés. Aucun profil type de jeunes n'a donc émergé de l'analyse. Toutefois, nous remarquons que le type de lien entre l'abuseur et la victime a un impact sur la façon dont les MTP sont vécus.

Tout d'abord, seront tour à tour présentées, de façon transversale, l'attribution des causes, la perception de contrôle, les perceptions face à l'abuseur (caractéristiques et sentiments actuels ressentis à son égard). Puis, nous présenterons la façon dont sont perçus les MTP en fonction du lien entre la victime et l'abuseur. Les éléments de discussion y seront intégrés afin d'alléger le texte. Aussi, des extraits de verbatim sont présentés pour illustrer les résultats.

\section{« COMMENT T'EXPLIQUES-TU CE QUE TU AS VÉCU?»}

\section{Résultats}

En ce qui concerne l'attribution des causes, d'une part, les participants et participantes ont nommé diverses causes. Des jeunes pouvaient même en nommer une variété, tout en demeurant cohérents dans leurs propos, pour expliquer un seul exemple de MTP. D'autre part, quelques participants et participantes ont eu de la difficulté à expliquer pourquoi les MTP s'étaient produits, ce qui reflète une certaine confusion. Cela rejoint les résultats d'études portant sur des victimes de MTP (DeRobertis, 2004) et d'abus sexuels (Feiring et Cleland, 2007; Feiring et al., 2002) qui montrent une difficulté à comprendre pourquoi elles ont été victimisées.

Causes individuelles. Tout d'abord, il est question des causes individuelles où, selon les jeunes interviewé(e)s, une personne directement impliquée dans l'épisode de violence, donc eux ou l'abuseur, est à l'origine des MTP.

Plus précisément, concernant l'abuseur, des jeunes vont directement le blâmer ou se déculpabiliser :

Ben il faisait ça, pour m'écœurer, pour faire son frais (se mettre en valeur), pour se penser bon devant ses amis. $(03)^{3}$

J'avais rien fait pourtant. (02)

D'autres perçoivent que l'abuseur a des problèmes de santé mentale ou de consommation, ou qu'il est atteint émotionnellement :

Elle a peut-être un problème dans sa tête. (02)

Ben mon père y prenait souvent d'la drogue, pis d'l'alcool. (04) 
Certain(e)s croient que l'abuseur ne les aime pas :

On aurait dit qu'elle m'haïssait là. (18)

Quelques-un(e)s croient que des émotions chez l'abuseur le poussent à être violent :

On dirait que c'était déjà une bulle de colère qui passait dès le matin. (07)

Puis, des participants et participantes pensent que l'abuseur souhaite les aider, mais qu'il s'y prend mal :

Plus il me traitait de noms, plus il pensait que j'allais me mettre au sport, au régime pis que j'allais arrêter de manger beaucoup, que j'allais maigrir. (12)

En ce qui concerne les causes attribuées aux jeunes eux-mêmes, deux catégories ont émergé. Premièrement, il y a plusieurs participants et participantes qui se reconnaissent une part de responsabilité, mais sans se tenir entièrement responsables. En fait, ils croient que d'autres facteurs ont contribué à la survenue des MTP. Par exemple, ce jeune mentionne qu'il avait effectivement insulté l'abuseur, mais que c'est ce dernier qui aurait interprété ses propos à tort en l'insultant à son tour et en le menaçant :

Je faisais rien que le niaiser un peu et il m'a dit ça de même. (03)

De plus, une jeune reconnaît qu'elle faisait de l'embonpoint. Cependant, elle nous précisait un peu plus loin dans l'entrevue que c'est son grand-père qui lui avait de la difficulté à accepter qu'elle était grosse et qui était alors dénigrant à son égard :

C'est parce que je pense que c'était à cause de ma grosseur. Quand j'étais petite, j'étais grosse. (12)

Cette jeune nous mentionne donc que son embonpoint a joué un rôle dans la survenue des MTP, mais que ce sont d'autres facteurs, tels l'intolérance de son grand-père à son égard, qui ont précipité les MTP.

Deuxièmement, il n'y a que quelques jeunes qui se blâment entièrement et qui perçoivent avoir précipité les MTP :

J'ai affronté du monde que j'aurais pas dû affronter. (10)

J'ai fait des conneries l'année passée. (14)

D'autres études portant sur des enfants et des adolescents et adolescentes victimes de violence notent elles aussi que peu se blâmaient pour les abus subis (e.g., Feiring et al., 2002; Morrow, 1991).

Causes familiales. Certains jeunes, victimes de MTP de source intrafamiliale ou extrafamiliale (par les pairs ou par des connaissances adultes), attribuent les MTP subis à des causes familiales. Premièrement, certains font allusion à la faiblesse du lien perçu entre eux et leur parent. En effet, un participant expliquait les MTP par la non-disponibilité de son parent au moment où il est né comparativement à un autre membre de la fratrie alors qu'un autre répondant mentionnait que les MTP étaient dus au fait de ne pas avoir été un enfant désiré au départ. De plus, un jeune attribuait les MTP à l'absence de liens de consanguinité, c.-à-d., par le fait de ne pas être le vrai enfant de son beau-père :

Peut-être quand ma demi-sœur est née, c'était sa fille pis pas moi. Il n'a jamais frappé ma sœur, mais moi oui [.. . ]. Il me regardait pas comme ça avant que ma sœur naisse. (05) 
Deuxièmement, d'autres croient qu'un événement stressant vécu par un membre de la famille, autre que par le jeune ou l'abuseur, peut avoir joué un rôle dans l'apparition des MTP :

Depuis que mon frère s'est suicidé, [. . . ] pis depuis ce temps-là mon père est comme perdu un peu là, pis c'est toujours de notre faute là. (06)

Troisièmement, la transmission intergénérationnelle de la violence est mentionnée. En effet, selon le participant, les MTP peuvent être expliqués par le fait que son parent ait subi de la maltraitance alors qu'il était enfant et qu'il reproduit cette violence à son tour :

Lui-même, ce qu'il a pu vivre, il va le faire vivre à ses enfants. Ce qu'il a vécu, il va nous le faire vivre à nous autres là aussi là! C'est pas pour rien que mon père est de même là, c'est parce qu'il veut nous faire vivre ce qu'il a vécu lui-même. (09)

Finalement, un jeune croit que des difficultés présentes au sein de la relation entre deux membres de la famille, ce qui l'exclut, expliquent les crises de colère importantes de son père à la maison :

Moi, je pense que mon père était tanné de ma mère. (13)

Causes liées à l'environnement. Selon des participants et participantes, des causes telles qu'une situation financière précaire chez l'abuseur ou encore des suggestions faites par des milieux institutionnels peuvent avoir joué un rôle dans la survenue des MTP :

Elle était paumée! Elle n'avait pas d'argent. (16)

Quand on était chez ma tante, moi pis mon frère, moi je suis partie en famille d'accueil pis mon frère est allé chez ma mère. Elle a comme eu, j'imagine, un choix! Tsé, je sais pas, le policier, je sais pas trop, le monde qui s'occupaient de la DPJ lui a dit : «Tu en prends un des deux enfants pis si ça va bien tu vas prendre l'autre. » Une affaire de même. (17)

\section{Discussion}

L'analyse met en lumière une variété d'explications causales, c.-à-d., que les participants et participantes attribuent les MTP à des causes individuelles, familiales ou liées à l'environnement. Nos résultats rejoignent, en partie, ceux de Gagné et al. (2010) qui montrent que les victimes de violence psychologique parentale font des attributions causales variées pour s'expliquer la violence. C'est aussi le cas chez des victimes d'abus sexuels (e.g., McGee, Wolfe et Olson, 2001). Cependant, notre classification des causes, plus complète, enrichit la littérature et nous permet de dépasser les réponses dichotomiques et d'aborder les nuances. Entre autres, nos résultats se distinguent de la proposition faite par Weiner (1986), et utilisée encore aujourd'hui, où les attributions sont soit internes (événement causé par ses propres caractéristiques) soit externes (événement causé par quelqu'un ou quelqu'une ou quelque chose). Aussi, notre classification nous semble plus riche que celle utilisée par Zinzow, Seth, Jackson, Niehaus et Fitzgerald (2010), où ils investiguaient le fonctionnement d'adultes ayant été victimes d'abus sexuels dans l'enfance (blâme de soi, blâme de l'abuseur ou blâme de la famille). De plus, d'autres auteurs classent l'attribution des causes obtenue à l'aide d'une question ouverte en fonction de trois catégories : (a) blâme de l'abuseur, (b) blâme de soi et (c) je ne sais pas (Feiring et Cleland, 2007). À la lumière de nos résultats, ces façons de procéder semblent simplifier la réalité et laisser de côté d'autres explications telles que les causes familiales et liées 
à l'environnement que les jeunes peuvent entretenir. Les auteurs pourraient donc s'inspirer de nos résultats pour mener des études quantitatives ou pour construire des questionnaires.

De plus, nos résultats se distinguent par le fait que nous avons précisé le blâme de soi et le blâme de l'abuseur. Par exemple, tel que mentionné plus tôt, un jeune pouvait blâmer sévèrement l'abuseur alors qu'un autre pouvait reconnaître que l'abuseur avait un problème de santé mentale. Cela va dans le même sens que Zinzow et ses collègues (2010) qui mentionnent que l'on ne devrait pas mesurer les attributions du blâme à l'aide d'un seul item, mais plutôt tenir compte des différents niveaux que peuvent prendre le blâme de soi et le blâme de l'abuseur.

Par ailleurs, il peut être positif de constater que peu de participants et participantes considèrent avoir provoqué les MTP. Au contraire, tout comme d'autres études portant sur des victimes de violence, nombreux sont ceux et celles qui ont nuancé leurs propos ou qui ont blâmé l'abuseur (e.g., Gagné et al., 2010; Kolko, Brown et Berliner, 2002). Ces résultats sont encourageants, car le blâme de soi est associé à divers symptômes de dysfonctionnement chez les victimes de violence (e.g., Barker-Collo, 2001; Feinauer et Stuart, 1996; Fortin, 2005; McGee et al., 2001; Steel, Sanna, Hammond, Whipple et Cross, 2004) alors que l'attribution externe du blâme (blâme de l'abuseur ou de l'entourage) est une variable à considérer dans l'étude de la résilience chez les enfants maltraités (Heller, Larrieu, D'Imperio et Boris, 1999). D'ailleurs, Valentine et Feinauer (1993), ayant étudié le discours de femmes victimes d'abus sexuels résilientes, avancent que le fait de reconnaître que l'abuseur a eu tort aide les victimes à surmonter les conséquences négatives liées aux abus.

Toutefois, il est possible que le blâme de soi semble moins fréquent chez les participants et participantes étant donné l'utilisation des questions ouvertes lors de l'entrevue (e.g., Feiring et al., 2002). En effet, Feiring et ses collègues (2002) notent que les attributions internes (blâme de soi) varient en fonction de la cueillette de données employée. Leur recherche, menée auprès de victimes d'abus sexuels, montre effectivement que les attributions internes spécifiques à l'abus sont quasi absentes lors de l'utilisation de questions ouvertes alors qu'elles sont plus fréquentes lorsque les participants et participantes doivent répondre aux items d'un questionnaire, soit l'Abuse Attribution Inventory.

Finalement, est-ce qu'externaliser le blâme serait leur façon de se défendre d'une certaine blessure narcissique? Rappelons-nous que les jeunes rencontrés étaient vulnérables et qu'ils avaient vécu des événements difficiles ayant pu les blesser. S'attribuer une partie du blâme serait peut-être trop menaçant puisque cela serait susceptible d'alimenter une certaine faille narcissique (Kohut, 1991). Cette hypothèse serait évidemment à investiguer éventuellement.

\section{«PENSAIS-TU AVOIR UN CERTAIN CONTRÔLE?»}

\section{Réponses négatives}

Résultats. Concernant la perception de contrôle, plusieurs jeunes indiquent ne pas avoir eu le contrôle durant l'épisode de MTP pour diverses raisons. En effet, ils sont nombreux à mentionner que, de leur point de vue, la situation de violence ou l'abuseur leur semblaient incontrôlables :

C'est sûr que t'as pas le contrôle sur un homme de 47 ans qui sait quoi faire pis qui sait tout quoi dire. (02) 
Parce que le monde ils ont le droit de parler là. Je peux pas leur enlever le droit de parler là. Même s'ils disent des trucs qui sont pas vrais, [.. .] je peux pas les empêcher de parler là. (18)

De plus, certaines et certains se sentaient impuissants et pris dans cette situation, alors que d'autres mentionnent qu'ils étaient trop jeunes pour faire quoi que ce soit. Quelques participantes et participants concluent qu'ils n'ont pas eu le contrôle puisque leurs stratégies d'adaptation adoptées pour faire face aux MTP n'ont pas apporté le changement souhaité :

J'ai essayé, mais ça a rien changé! (14)

Aussi, quelques-un(e)s ne savaient pas quoi faire. Finalement, un participant perçoit ne pas avoir eu le contrôle puisqu'il reconnaît avoir eu besoin de l'aide d'un tiers pour parvenir à ses fins.

Discussion. Ces exemples de perte de contrôle montrent à quel point les MTP ont entraîné une souffrance importante chez les victimes. D'ailleurs, d'autres auteurs notent aussi que les victimes de violence psychologique avaient l'impression d'être démunis et impuissants (Gagné et al., 2010; Joseph et al., 2006). Ces résultats doivent être considérés sérieusement puisque des études indiquent que l'impuissance et la perception de ne pas avoir le contrôle sont associées, entre autres, à la détresse psychologique (e.g., Dekel, Mandl et Solomon, 2011), à l'émergence de symptômes dépressifs (e.g., Ceyhan et Ceyhan, 2009; Jang, Chiriboga, Kim et Rhew, 2010) et à une faible estime de soi (Hazzard, 1993).

\section{Réponses positives}

Résultats. D'autres jeunes mentionnent avoir eu le contrôle pour différentes raisons, ce qui montre que le concept ne doit pas être traité de façon dichotomique (Fournier et Jeanrie, 2003). Premièrement, des participants et participantes croient avoir eu le contrôle car ils ont réussi à avoir un effet sur la situation de violence ou sur l'abuseur :

Ben je lui parlais pis elle m'écoutait pour une fois là. (10)

Deuxièmement, des adolescents et adolescentes reconnaissent que c'est la consommation de substances qui leur a donné l'illusion d'être en contrôle :

J'avais l'impression d'être plus puissant que lui parce que j'avais consommé pis fumé. (10)

Puis, des répondants et répondantes notent avoir eu le contrôle étant donné la confiance qu'ils ont en général en eux-mêmes et en leurs capacités.

Discussion. Bien que ces résultats puissent être encourageants étant donné que percevoir avoir le contrôle serait un important facteur de protection chez les victimes de violence (e.g., Bolger et Patterson, 2001; Henry, 1999; O’Neill et Kerig, 2000) et associé à un faible niveau de détresse (Frazier et al., 2011), ils doivent être interprétés avec prudence. En effet, des auteurs notent que ce n'est pas nécessairement favorable (Fournier et Jeanrie, 2003). La perception d'avoir le contrôle pourrait plutôt refléter l'incapacité chez une personne à reconnaître ses limites. Par exemple, chez un jeune qui affirme avoir le contrôle sur tout ce qui l'entoure ou chez un autre qui dit avoir la fausse impression d'être invincible étant donné la quantité de drogues et d'alcool consommées. 


\section{PENSÉES ET SENTIMENTS ENVERS L'ABUSEUR}

\section{«Que penses-tu de celui ou celle qui t’a fait ça?»}

Lorsque les participants et participantes ont été invités à nous dire ce qu'ils pensent actuellement de l'abuseur, la majorité ont nommé des caractéristiques négatives. En effet, des jeunes ont traité l'abuseur de noms ou ont été dénigrants à son égard. De plus, plusieurs trouvent que l'abuseur est « méchant» :

Il est vraiment agressif là. (18)

Aussi, certains et certaines ont critiqué ce que l'abuseur avait fait dans le passé, que ce soit lié ou non à l'épisode de violence :

Moi je trouve juste qu'il a pas pris la bonne façon de me le dire, qu'il aurait pu faire mieux. (12)

D'autres ont plutôt mentionné que l'abuseur est en difficulté, qu'il a un trouble mental ou qu'il a besoin d'aide :

Pis je suis sûre qu'il a une maladie mentale quelconque soit dépression, bipolaire des choses de-même. (06)

Finalement, d'autres caractéristiques, moins nommées, telles le fait d'être immature ou de se sentir supérieur ont aussi été attribuées à l'abuseur.

Par ailleurs, quelques jeunes ont nommé des caractéristiques positives lorsqu'ils parlaient de l'abuseur :

Je pense que c'est un bon gars quand il veut. (09)

C'est la meilleure mère qu'on ne peut pas avoir. (10)

\section{«Que ressens-tu actuellement à l'égard de cette personne? »}

Concernant les sentiments actuels envers l'abuseur, encore une fois, les sentiments négatifs sont nombreux, alors que d'autres se disent indifférents et que quelques-un(e)s disent aimer l'abuseur. Plus précisément, plusieurs se disent choqués alors que d'autres nomment la confusion ou des sentiments mélancoliques tels que la tristesse et la pitié, ainsi que la peur. On peut noter que parmi les adolescentes et adolescents qui ont dit être choqués, 10 entretenaient des sentiments hostiles ou désiraient se venger, ce qui rejoint les résultats de Joseph et al. (2006) :

$\mathrm{Si}$ on pouvait la retrouver, on la ferait payer cher! (02)

Je le revois, je lui casse les deux dents, les deux jambes et les deux bras. (03)

J'ai de la haine dans le fond. (09)

\section{Discussion}

Dans l'ensemble, tout comme Joseph et al. (2006), notre étude montre qu'il y a des jeunes qui parviennent à nuancer leurs propos, c.-à-d., qu'ils expriment des sentiments mixtes, alors qu'il y en a d'autres qui sont encore en colère et qui dénigrent à leur tour l'abuseur. Kolko et al. (2002), s'étant penché sur des enfants et adolescents et adolescentes victimes d'abus physiques et/ou sexuels, montrent aussi que les victimes ressentaient des sentiments soit positifs ou négatifs, ou encore, des sentiments mixtes à l'égard de l'abuseur. 
Par ailleurs, ces résultats suggèrent deux pistes de réflexion. D'une part, on remarque que les victimes peuvent être affectées par les MTP pendant une longue période de temps. En effet, certains jeunes, qui avaient été rabaissés par un membre de la famille depuis des années déjà, nous décrivaient sévèrement l'abuseur et entretenaient encore des sentiments hostiles à son égard. Pour d'autres, la peur était encore présente et ils redoutaient de rencontrer à nouveau l'abuseur. D'autre part, la dureté des propos de certains participants et participantes à l'égard de l'abuseur est frappante. Il est possible que, pour certain(e)s, nous parler avec violence de l'abuseur leur permettait de ventiler leur colère. Toutefois, il est tout aussi possible que certains de ces jeunes soient à risque d'adopter éventuellement des comportements violents à l'égard de l'abuseur. D'ailleurs, tel que le présentent Bêty et al. (2011), des jeunes avaient réagi avec violence lors des MTP. Il est donc possible que de jeunes victimes puissent adopter à leur tour des comportements violents (Celano, Hazzard, Campbell et Lang, 2002).

\section{PERCEPTIONS DES MTP EN FONCTION DES TYPES DE LIEN ENTRE LA VICTIME ET L'ABUSEUR}

Le tableau 2 présente les perceptions des MTP en fonction des types de lien entre la victime et l'abuseur. Nous y retrouvons la violence intrafamiliale, présente dans 15 des 36 incidents critiques recueillis, et la violence extrafamiliale qui comprend la violence par les pairs (14 des 36 incidents critiques), la violence par les intervenants et intervenantes (4 des 36 incidents critiques) et la violence par des connaissances adultes ( 3 des 36 incidents critiques). Afin de simplifier l'analyse, les catégories de certaines perceptions sont modifiées. Par exemple, lorsque les participants et participantes nommaient différentes causes pour s'expliquer les MTP subis, nous considérons qu'ils « nuancent » leurs propos.

\section{Violence intrafamiliale}

Douze des 19 incidents critiques de MTP les plus stressants à vie constituent de la violence intrafamiliale, ce qui suggère que la violence intrafamiliale engendre une souffrance importante et marquante chez les enfants et les adolescents et adolescentes. Dans l'ensemble, on remarque que les jeunes nuancent davantage l'attribution des causes lorsqu'il s'agit de violence intrafamiliale, c.-à-d., qu'ils nomment plusieurs causes (individuelles, familiales ou externes) ou qu'ils considèrent que des difficultés chez l'abuseur l'ont mené à être violent. De plus, leurs propos sont aussi nuancés lorsqu'ils sont invités à nous parler de l'abuseur et des sentiments ressentis à son égard. En effet, les deux tiers des victimes de violence intrafamiliale parvenaient à attribuer des caractéristiques positives à l'abuseur et $40 \%$ ressentent actuellement des sentiments positifs envers l'abuseur (tableau 2). De plus, c'est seulement chez les victimes de violence intrafamiliale que l'on retrouve des descriptions de l'abuseur strictement positives.

Il est compréhensible que les participants et participantes aient tendance à nuancer davantage leurs propos lorsqu'il est question de violence intrafamiliale. En effet, comme le mentionnent Kolko et al. (2002), blâmer sévèrement son parent serait presque menaçant parce qu'en lui attribuant tous les torts, le jeune serait pris avec sa perception que son parent est mauvais ou que ce dernier ne l'aime pas. En nuançant, il se protège et entretient l'idée que, malgré tout, son parent est bon et que d'autres facteurs font en sorte qu'il a été violent. Toutefois, ces résultats peuvent aussi être expliqués par le fait que le style parental a un effet sur le style 


\section{Tableau 2}

Perceptions en fonction du type de lien entre la victime et l'abuseur (\%)

\begin{tabular}{|c|c|c|c|c|}
\hline \multirow[t]{2}{*}{ Perceptions } & \multirow{2}{*}{$\begin{array}{c}\text { Violence } \\
\text { intrafamiliale } \\
n=15\end{array}$} & \multicolumn{3}{|c|}{ Violence extrafamiliale } \\
\hline & & $\begin{array}{c}\text { Par les pairs } \\
\quad n=14\end{array}$ & $\begin{array}{c}\text { Par les } \\
\text { intervenant(e)s } \\
n=4\end{array}$ & $\begin{array}{c}\text { Par des } \\
\text { connaissances } \\
\text { adultes } \\
n=3\end{array}$ \\
\hline \multicolumn{5}{|l|}{ Attribution des causes } \\
\hline Blâme de l'abuseur & 20,0 & 50,0 & 100,0 & 33,3 \\
\hline Nuance & 73,3 & 7,1 & 0,0 & 66,6 \\
\hline Blâme de soi & 6,7 & 14,3 & 0,0 & 0,0 \\
\hline Croyance de ne pas être aimé(e) & 0,0 & 21,4 & 0,0 & 0,0 \\
\hline Ne sait pas & 0,0 & 7,1 & 0,0 & 0,0 \\
\hline \multicolumn{5}{|l|}{ Perception de contrôle } \\
\hline Non & 66,7 & 71,4 & 100,0 & 33,3 \\
\hline Oui & 26,7 & 14,3 & 0,0 & 66,7 \\
\hline Non interprétable & 6,7 & 14,3 & 0,0 & 0,0 \\
\hline \multicolumn{5}{|l|}{ Description de l'abuseur } \\
\hline Dénigrante & 33,3 & 50,0 & 100,0 & 0,0 \\
\hline Nuancée & 53,3 & 42,9 & 0,0 & 100,0 \\
\hline Positive & 13,3 & 0,0 & 0,0 & 0,0 \\
\hline Non interprétable & 0,0 & 7,1 & 0,0 & 0,0 \\
\hline \multicolumn{5}{|l|}{ Sentiments actuels envers l'abuseur" } \\
\hline Peur & 13,3 & 0,0 & 0,0 & 0,0 \\
\hline Confusion & 13,3 & 0,0 & 0,0 & 33,3 \\
\hline Choqué & 40,0 & 42,9 & 50,0 & 66,7 \\
\hline Hostilité & 26,7 & 28,6 & 50,0 & 33,3 \\
\hline Sentiments mélancoliques & 6,7 & 0,0 & 0,0 & 33,3 \\
\hline Indifférence & 13,3 & 35,7 & 25,0 & 33,3 \\
\hline Positifs & 40,0 & 14,3 & 0,0 & 0,0 \\
\hline
\end{tabular}

${ }^{*}$ Le total peut être de plus de $100 \%$. 
d'attribution de l'enfant. En effet, des études indiquent que des comportements parentaux tels qu'induire la honte, la culpabilité et l'anxiété chez l'enfant, ce qui s'apparente aux MTP, ont un impact sur les cognitions de l'enfant (Garber et Flynn, 2001). En se faisant blâmer durant des années par des figures significatives, il est possible que l'enfant intériorise ces verbalisations et en vienne à nuancer ses propos pour banaliser l'impact des MTP (Garber et Flynn, 2001; Logan, Walker, Jordan et Leukefeld, 2006).

Par ailleurs, les résultats mettent aussi en relief la souffrance engendrée par la violence intrafamiliale. En effet, deux tiers des victimes mentionnent ne pas avoir eu le contrôle lors des MTP (tableau 2).

\section{Violence extrafamiliale par les pairs}

Ce type de violence se distingue des autres sur quelques éléments uniquement. On note que la perception « croire ne pas être aimé » pour expliquer les MTP est seulement présente chez les victimes de violence extrafamiliale par les pairs. Cela n'est pas surprenant chez un échantillon juvénile puisque c'est à l'adolescence que les pairs jouent un rôle central et «c'est vers eux que se tourne l'adolescent lorsqu'il prend ses distances par rapport à ses parents » (Cloutier et Drapeau, 2008, p. 194). Il est donc fort probable que les adolescents et adolescentes se soucient beaucoup de l'opinion des pairs et du fait d'être apprécié par ces derniers.

\section{Violence extrafamiliale par les intervenants et intervenantes}

La violence par les intervenants et intervenantes semble se distinguer de la violence intrafamiliale sur différents points. Toutefois, il faut interpréter ces résultats avec prudence étant donné le peu d'épisodes recueillis quant à ce type de violence. Nous trouvons tout de même pertinent de faire part des résultats afin de rendre justice à la parole des jeunes.

Tout d'abord, la totalité des victimes de ce type de violence blâment l'abuseur (tableau 2). Le fait de blâmer l'abuseur de ce type de violence peut être expliqué par différentes raisons. En premier lieu, les jeunes ayant raconté ce type d'épisode de violence devaient avoir des attentes par rapport aux services rendus par les employés et employées du réseau public. Or, parmi les exemples émis par les jeunes, être rabaissé par un agent de la paix ou par un employé du Centre Jeunesse, ou encore, subir l'indifférence d'un professionnel de la santé peut avoir augmenté le sentiment d'être injustement traité et contribué au blâme de l'abuseur. Cette hypothèse rejoint, en partie, ce que concluent Valle et Silovsky (2002) quant à la violence physique parentale. Ils indiquent effectivement que les enfants ont tendance à blâmer davantage leur abuseur lorsque les comportements violents dépassent largement ce qui est attendu au sein de la relation parent-enfant. En deuxième lieu, les jeunes rencontrés reçoivent des services du Centre Jeunesse depuis plusieurs mois déjà. Il est possible qu'ils étaient déjà désillusionnés quant aux services rendus et irrités par ces derniers, ce qui peut aussi avoir contribué au blâme de l'abuseur.

Concernant la perception de contrôle, il est intéressant de constater que l'ensemble des victimes de violence par les intervenants et intervenantes perçoivent ne pas avoir eu le contrôle (tableau 2). Cela peut s'expliquer par le fait que les professionnels et professionnelles représentent des figures d'autorité pour le jeune, ce qui semble l'amener à se sentir impuissant. 
Finalement, nos résultats montrent que l'ensemble des victimes de violence par les intervenants et intervenantes dénigrent l'abuseur et que l'hostilité tend à être plus présente chez les victimes de violence extrafamiliale par les intervenants et intervenantes.

\section{Violence extrafamiliale par des connaissances adultes}

Ce type de violence est plus difficile à interpréter étant donné la diversité des trois épisodes recueillis. Il ne sera donc pas analysé en profondeur comme les précédents. Par contre, afin de montrer l'ampleur des MTP rapportés par les jeunes rencontrés, nous trouvons pertinent de décrire brièvement ces trois épisodes de violence.

Le premier épisode concerne une jeune fille qui a été victime d'un homme assez âgé qui l'a manipulée et exploitée pour parvenir à ses fins, c.-à-d., pour que l'adolescente lui paie la drogue qu'il lui avait donnée. L'adolescente raconte qu'elle sentait toute l'emprise qu'avait cet homme sur elle et que sans qu'il ne parle, elle savait ce qu'elle avait à faire pour lui « payer » sa drogue. La jeune s'attribuait une partie du blâme puisque lorsqu'elle avait croisé l'homme, elle ne se sentait pas bien et elle avait décidé d'aller vers lui afin de consommer. Toutefois, elle reconnaît aussi que cet homme est un manipulateur qui sait quoi dire et quoi faire pour obtenir ce qu'il veut. Elle ajoutait ressentir du dégoût à son égard. De plus, la participante critiquait ce que l'homme lui avait fait. Par ailleurs, durant cet épisode, l'adolescente avait de la peine et se sentait impuissante.

Le deuxième épisode fait référence à un jeune qui a subi des menaces de mort de la part d'un homme qui s'exhibait près de lui et de sa copine. Le jeune s'expliquait cet événement par le fait que l'homme devait avoir un problème de santé mentale et qu'il devait s'être senti intimidé par le jeune qui lui demandait de s'en aller. Bien que les émotions rapportées par le participant soient la peine, la peur et la colère, le jeune a perçu avoir le contrôle puisqu'il n'a pas hésité à demander à l'exhibitionniste de partir et à appeler la police pour faire en sorte que l'homme quitte les lieux. En pensant à cet homme, le participant ressentait de la peine et de la colère. Il disait que, pour lui, cet homme était malade et qu'il méritait donc de se retrouver dans un hôpital psychiatrique ou en prison.

Finalement, le troisième épisode recueilli a été raconté par un jeune qui avait subi des menaces de mort de la part de son supérieur pour qui il vendait de la drogue puisqu'il lui devait de l'argent depuis un certain temps déjà. Le jeune blâmait son patron d'interpréter à tort la situation. Aussi, le participant blâmait son patron d'être aussi exigeant et de ne penser qu'à son argent. Suite aux menaces, le jeune reconnait qu'il avait très peur, qu'il était anxieux et en colère et qu'il avait envie de se venger. Bien qu'il ait été apeuré au départ, le jeune mentionne qu'il s'était ressaisi et qu'il avait alors perçu avoir du contrôle. En effet, le jeune avait confiance en ses capacités et avait décidé de s'expliquer avec son supérieur. Lorsqu'il a été invité à nous parler de ce dernier, le jeune avait nuancé ses propos en mentionnant que d'un côté, c'est une bonne personne, alors que de l'autre côté, il lui reprochait d'accorder autant d'importance à l'argent. Finalement, le jeune se disait indifférent à son égard.

Les trois exemples de violence extrafamiliale par des connaissances adultes mettent donc en lumière le contexte dans lequel vivent les jeunes rencontrés, la consommation et la violence étant effectivement très présentes. De plus, ces trois exemples montrent à eux seuls ce qui a été mentionné plus tôt, c.-à-d., que les 
participants et participantes présentent une variété de réponses affectives et cognitives lorsqu'ils subissent des MTP.

\section{Retour sur les quatre types de lien entre la victime et l'abuseur}

L'analyse met en lumière que la violence intrafamiliale est vécue différemment par les victimes. En effet, ces dernières nuancent leurs propos, attribuent les MTP à plusieurs causes et accordent des caractéristiques tant positives que négatives à l'abuseur. De plus, cette violence " frappe », car la majorité des incidents critiques recueillis comme les plus stressants étaient en fait des épisodes de violence intrafamiliale, lesquels comportent des enjeux plus complexes et délicats à gérer au niveau des impacts cliniques et identitaires.

\section{CONCLUSION}

\section{Forces et limites}

Cette étude présente différentes forces. Il s'agit tout d'abord d'une des premières recherches à aborder directement les MTP subis au sein de diverses relations sociales avec des adolescents et adolescentes à très haut risque. En outre, notre approche assure une richesse du propos. En effet, en ayant demandé aux jeunes de nous raconter deux exemples de MTP, nous constatons qu'ils ont réagi différemment à l'un ou l'autre des deux exemples, ce qui permet une description complexe tenant compte du contexte. Aussi, notre étude met en lumière une violence peu abordée dans les écrits et sur le terrain, et qui aurait intérêt à être documentée davantage, soit la violence extrafamiliale par les intervenants et intervenantes. De plus, notre analyse qualitative a permis de préciser les catégories telles que le blâme de soi ou le blâme de l'abuseur. Finalement, demander aux participants et participantes de nous raconter un exemple récent de MTP ou encore se limiter aux 3 derniers mois nous a permis d'avoir accès à des souvenirs et perceptions possiblement plus précis, ce qui pallie aux limites souvent associées aux études rétrospectives et menées auprès d'adultes pour comprendre la violence subie en enfance.

Cette étude comporte quelques limites. Premièrement, l'échantillon comporte seulement 6 filles versus 13 garçons. Il serait intéressant de réaliser une étude similaire auprès d'un échantillon comprenant davantage de filles afin d'étudier les différences ou spécificités de genre. Deuxièmement, il est difficile de départager les MTP des autres types de violence. Dans le cadre de notre étude, des jeunes racontaient parfois des exemples où il y avait à la fois des MTP et un abus sexuel ou physique. Il pourrait être pertinent d'investiguer à nouveau les MTP en recueillant des exemples qui réfèrent uniquement aux MTP ou d'élaborer une approche soulignant ce type de cumul. Troisièmement, puisque les enfants sont moins enclins à nommer des causes internes (blâme de soi) lorsqu'on formule des questions ouvertes (Feiring et Cleland, 2007; Feiring et al., 2002; Kolko et al., 2002), l'utilisation d'un questionnaire standardisé ajouté à l'entrevue nous aurait permis de faire une évaluation exhaustive des attributions spécifiques aux MTP (Cohen et Mannarino, 2002). Finalement, les exemples racontés étaient parfois compliqués à analyser puisque la situation était chronique ou qu'il pouvait y avoir plus d'un abuseur. Il était alors difficile d'investiguer les perceptions et les émotions qui fluctuaient dans le temps et d'aborder les sentiments ressentis à l'égard de plus d'un abuseur. Parfois, les sentiments ressentis à l'égard des deux abuseurs étaient fort différents. 


\section{Retombées pratiques}

Nos résultats mettent en relief la souffrance engendrée par les MTP qui peuvent parfois être banalisés par les parents, les pairs, les enseignants et enseignantes et les intervenants et intervenantes. Étant donné leurs impacts destructeurs, les MTP présents au sein des diverses relations sociales auraient avantage à faire l'objet d'un questionnement plus systématique au sein des divers processus d'évaluation et d'intervention cliniques, et ce, particulièrement dans les Centres Jeunesse.

Tel que mentionné, les participantes et participants rencontrés se disaient soulagés d'avoir pu parler des MTP subis. Pour certains et certaines, c'était la première fois qu'ils prenaient le temps de réfléchir et de comprendre ce qui s'était passé. Donc, il peut être fort pertinent de prendre le temps de questionner la victime sur ce qui s'est passé en abordant les différentes variables cognitives qui ont été investiguées dans la présente étude. Face à un jeune qui tend à se blâmer sévèrement, différentes stratégies d'intervention pourraient être utilisées afin de l'amener à nuancer davantage ses idées. Aussi, un soutien pourrait être offert pour l'amener à poser ses limites et à ne pas tolérer tout ce qu'il subit.

Les MTP sont vécus différemment en fonction du type de relation entretenue avec l'abuseur. Lorsqu'il s'agit de violence intrafamiliale, les intervenants et intervenantes devraient donc avoir comme objectif de diminuer le blâme de soi, s'il y a lieu, sans nécessairement tenter d'augmenter le blâme de l'abuseur (Cohen et Mannarino, 2002). Au contraire, respecter les jeunes qui désirent conserver une perception nuancée ou positive de son parent serait plus utile (Kolko et al., 2002). C'est au fil du temps que l'adolescent ou l'adolescente pourra faire le deuil du bon parent qu'il aurait souhaité avoir.

Les jeunes victimes de violence extrafamiliale par les pairs méritent aussi d'obtenir un soutien important de leur entourage. En effet, à l'adolescence, percevoir ne pas être accepté par les autres jeunes est susceptible d'engendrer des conséquences au plan de la santé mentale puisqu'à cet âge, les pairs jouent un rôle central (Cloutier et Drapeau, 2008).

Lorsqu'il s'agit de violence extrafamiliale par les intervenants et intervenantes, qui est souvent négligée dans la littérature et que notre étude permet d'analyser, les intervenants et intervenantes pourraient alors soutenir les jeunes qui ressentent un niveau de colère important et qui blâment l'abuseur. Cette colère pourrait être entendue, mais elle ne devrait pas être encouragée. Alimenter cette colère, surtout chez des jeunes impulsifs, auraient pour effet d'augmenter le risque de vengeance (Celano et al., 2002). Par ailleurs, les institutions concernées devraient revoir et questionner les divers processus censés s'assurer qu'aucun épisode de violence extrafamiliale par les intervenants et intervenantes ne soit vécu par les jeunes. Cela pourrait se faire, notamment, en sensibilisant les intervenants et intervenantes aux conséquences associées aux MTP.

\section{Perspectives d'intervention et de recherche}

Cette recherche ouvre la voie à des projets d'intervention et de recherche. En effet, étant donné la souffrance des victimes identifiée dans nos résultats, des approches de prévention en matière de MTP devraient être développées et mises sur pied dans les écoles, les Centre Jeunesse et les autres institutions touchant les jeunes. De plus, des études quantitatives pourraient donner suite à la présente étude en investiguant, auprès d'un large échantillon, les perceptions liées aux MTP à l'aide d'outils standardisés qu'il faudrait d'abord développer. Il serait alors possible d'évaluer si les variables subjectives expliquent la relation déjà soulevée 
dans la littérature entre les MTP et des problèmes de santé mentale. Finalement, réaliser cette même étude qualitative auprès de populations différentes pourrait nous mener à étudier des variations entre leur façon de vivre les MTP. Par exemple, il serait pertinent de reprendre cette étude auprès de jeunes provenant de différentes populations cliniques ou auprès d'une population non clinique et également d'analyser des incidents de violence en contexte de couple.

\section{NOTES}

1. Les Centres Jeunesse regroupent « les centres de protection de l'enfance et de la jeunesse (CPEJ), les points de services de CPEJ, les centres de réadaptation pour jeunes en difficulté d'adaptation et les centres de réadaptation pour jeunes mères en difficulté d'adaptation » (Ministère de la Santé et des Services sociaux du Québec, 2004).

2. «Les centres de réadaptation ont pour mission d'offrir des services d'adaptation, ainsi que de réadaptation et d'intégration sociale, à des [jeunes mineurs] qui, en raison [. . .] de leurs difficultés d'ordre comportemental, psychosocial ou familial, ou à cause de leur alcoolisme ou autre toxicomanie, requièrent de tels services, de même que des services d'accompagnement et de support à leur entourage » (Ministère de la Santé et des Services sociaux du Québec, 2004).

3. Le chiffre entre parenthèses correspond au numéro attribué au participant ou à la participante afin d'assurer la confidentialité.

\section{RÉFÉRENCES}

Anton, B. S. (2010). Proceedings of the American Psychological Association for the legislative year 2009: Minutes of the annual meeting of the Council of Representatives and minutes of the meetings of the Board of Directors. American Psychologist, 65(5), 385-475. doi: 10.1037/a0019553

Bardin, L. (1991). L'analyse de contenu. Paris : Presses universitaires de France.

Barker-Collo, S. L. (2001). Adult reports of child and adult attributions of blame for childhood sexual abuse: Predicting adult adjustment and suicidal behaviors in females. Child Abuse \& Neglect, 25, 1329-1341. doi:10.1016/ S0145-2134(01)00278-2

Beck, J. S. (1995). Cognitive therapy: Basics and beyond. New York, NY : Guilford Press.

Bêty, C. (2012). Les mauvais traitements psychologiques présents dans les relations sociales des adolescents ayant fait une tentative de suicide et recevant les services d'un Centre Jeunesse (Thèse de doctorat inédite). Université Laval, Québec, Québec.

Bêty, C., Lavoie, F. et Nadeau, D. (2011, Automne). Stratégies d'adaptation d'adolescents lors de mauvais traitements psychologiques. Sociétés et Jeunesses en Difficulté, 12.

Bifulco, A., Moran, P. M., Baines, R., Bunn, A. et Stanford. K. (2002). Exploring psychological abuse in childhood : II. Association with other abuse and adult clinical depression. Bulletin of the Menninger Clinic, 66(3), 241-258. doi : 10.1521/bumc.66.3.241.23366

Bolger, K. E. et Patterson, C. J. (2001). Pathways from child maltreatment to internalizing problems: Perceptions of control as mediators and moderators. Development and Psychopathology, 13, 913-940.

Brassard, M. R. et Donovan, K. L. (2006). Defining psychological maltreatment. Dans M. M. Feerick, J. F. Knuston et P. K. Trickett (dir.), Child abuse and neglect: Definitions, classifications, and a framework for research (pp. 151197). Baltimore, MD: Paul H Brookes.

Celano, M., Hazzard, A., Campbell, S. K. et Lang, C. B. (2002). Attribution retraining with sexually abused children: Review of techniques. Child Maltreatment, 7(1), 65-76. doi: 10.1177/1077559502007001006

Ceyhan, A. et Ceyhan, E. (2009). Relationship between early separation anxiety and depressive symptoms: The mediating role of locus of control and problem solving skills. Pakistan Journal of Social and Clinical Psychology, 7(1), 3-17.

Clément, M.-È., Chamberland, C., Côté, L., Dubeau, C. et Beauvais, B. (2005). La violence familiale dans la vie des enfants du Québec. Québec : Institut de la statistique du Québec.

Cloutier, R. et Drapeau, S. (2008). Psychologie de l'adolescence. Montréal, QC : Gaëtan Morin. 
Cohen, J. A. et Mannarino, A. P. (2002). Addressing attributions in treating abused children. Child Maltreatment, 7(1), 81-84.

Creswell, J. W. (2007). Qualitative inquiry and research design: Choosing among five approaches (2 $2^{\mathrm{e}}$ ed.). Thousand Oaks, CA : Sage.

Dekel, S., Mandl, C. et Solomon, Z. (2011). Shared and unique predictors of post-traumatic growth and distress. Journal of Clinical Psychology, 67(3), 241-252. doi: 10.1002/jclp.20747

DeRobertis, E. M. (2004). The impact of long-term psychological maltreatment by one's maternal figure: A study of the victim's perspective. Journal of Emotional Abuse, 4(2), 27-51. doi : 10.1300/J135v04n02_02

Deslauriers, J.-P. (1991). Recherche qualitative : guide pratique. Montréal, QC : McGraw- Hill.

Feinauer, L. L. et Stuart, D. A. (1996). Blame and resilience in women sexually abused as children. American Journal of Family Therapy, 24(1), 31-40. doi : 10.1080/01926189508251014

Feiring, C. (2005). Emotional development, shame, and adaptation to child maltreatment. Child Maltreatment, 10(4), 307-310. doi: 10.1177/1077559505281307

Feiring, C. et Cleland, C. (2007). Childhood sexual abuse and abuse-specific attributions of blame over 6 years following discovery. Child Abuse \& Neglect, 31, 1169-1186. doi: 10.1016/j.chiabu.2007.03.020

Feiring, C., Taska, L. et Chen, K. (2002). Trying to understand why horrible things happen: Attribution, shame, and symptom development following sexual abuse. Child Maltreatment, 7(1), 25-39. doi: 10.1177/1077559502007001003

Flanagan, J. C. (1954). The critical incident technique. Psychological Bulletin, 51(4), 327-357. doi: 10.1037/h0061470

Fortin, A. (2005). Le point de vue de l'enfant sur la violence conjugale à laquelle il est exposé. Rapport final de recherche. Montréal, QC : Centre de recherche interdisciplinaire sur la violence familiale et la violence faite aux femmes.

Fournier, G. et Jeanrie, C. (2003). Locus of control : Back to basics. Dans S. J. Lopez et C. R. Snyder (dir.), Positive psychological assessment: A handbook of models and measures (pp. 139-154). Washington, DC : American Psychological Association.

Frazier, P., Keenan, N., Anders, S., Perera, S., Shallcross, S. et Hintz, S. (2011). Perceived past, present, and future control and adjustment to stressful life events. Journal of Personality and Social Psychology, 100(4), 749-765. doi: $10.1037 / \mathrm{a} 0022405$

Frechtling, J. (2002). The 2002 user friendly handbook for project evaluation. Arlington, VA : National Science Foundation.

Gagné, M.-H., Drapeau, S., Melançon, C., Saint-Jacques, M.-C. et Lépine, R. (2007). Links between parental psychological violence, other family disturbances, and children's adjustment. Family Process, 46(4), 523-542. doi : $10.1111 / \mathrm{j} .1545-5300.2007 .00230 \mathrm{x}$

Gagné, M.-H., Melançon, C., Pouliot-Lapointe, J., Lavoie, F. et Roy, M. (2010). Violence psychologique des parents rapportée par des jeunes à un service d'aide anonyme. Revue canadienne de santé mentale communautaire, 29(2), 79-98.

Garber, J. et Flynn, C. (2001). Predictors of depressive cognitions in young adolescents. Cognitive Therapy and Research, 25(4), 353-376. doi: 10.1023/A :1005530402239

Gençoz, F., Vatan, S., Walker, R. et Lester, D. (2008). A brief research note: Helplessness, hopelessness, and haplessness as predictors of suicidal ideation: A cross-cultural study. Journal of Death and Dying, 57(3), 315-318. doi: 10.2190/OM.57.3.f

Giles, D. C. (2002). Advanced research methods in psychology. New York, NY: Psychology Press/Routledge.

Hazzard, A. (1993). Trauma-related beliefs as mediators of sexual abuse impact in adult women survivors: A pilot study. Journal of Child Sexual Abuse: Research, Treatment, \& Program Innovations for Victims, Survivors, \& Offenders, 2(3), 55-69. doi: 10.1300/J070v02n03_04

Heller, S. S., Larrieu, J. A., D'Imperio, R. et Boris, N. W. (1999). Research on resilience to child maltreatment: Empirical considerations. Child Abuse \& Neglect, 23(4), 321-338.

Henry, D. L. (1999). Resilience in maltreated children : Implications for special needs adoption. Child Welfare League of America, 78(5), 519-540.

Hurley, J. C. et Underwood, M. K. (2002). Children's understanding of their research rights before and after debriefing: Informed assent, confidentiality, and stopping participation. Child Development, 73(1), 132-143. doi: $10.1111 / 1467-8624.00396$ 
Institut de la statistique du Québec. (2010). Estimation de la population des régions administratives, $1^{\text {er }}$ juillet des

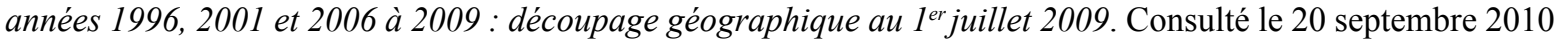
à : http ://www.stat.gouv.qc.ca/donstat/societe/demographie/dons_regnl/regional/ra_total.htm

Jang, Y., Chiriboga, D. A., Kim, G. et Rhew, S. (2010). Perceived discrimination, sense of control, and depressive symptoms among Korean American older adults. Asian American Journal of Psychology, 1(2), 129-135. doi: $10.1037 / \mathrm{a} 0019967$

Joseph, S., Govender, K. et Bhagwanjee, A. (2006). "I can't see him hit her again, I just want to run away ... hide and block my ears": A phenomenological analysis of a sample of children's coping responses to exposure to domestic violence. Journal of Emotional Abuse, 6(4), 23-45. doi: 10.1300/J135v06n04_02

Klomek, A. B., Marroco, F., Kleinman, M., Schonfeld, I. S. et Gould, M. S. (2007). Bullying, depression, and suicidality in adolescents. American Academy of Child and Adolescent Psychiatry, 46(1), 40-48. doi:10.1097/01. chi.0000242237.84925.18

Kohut, H. (1991). Le soi : la psychanalyse des transferts narcissiques. Paris : Presses universitaires de France.

Kolko, D. J., Brown, E. J. et Berliner, L. (2002). Children's perceptions of their abusive experience : Measurement and preliminary findings. Child Maltreatment, 7(1), 42-55. doi: 10.1177/1077559502007001004

Krug, E. G., Dahlberg, L. L., Mercy, J. A., Zwi, A. et Lozano-Ascencio, R. (2002). Rapport mondial sur la violence et la santé : Résumé. Genève, Suisse : Organisation mondiale de la santé.

Laperrière, A. (1994). Les critères de scientificité des méthodes qualitatives. Dans Colloque du Conseil québécois de la recherche sociale (dir.), Les méthodes qualitatives en recherche sociale : problématiques et enjeux: actes du Colloque du Conseil québécois de la recherche sociale tenu à Rimouski le 17 mai 1993 (pp.45-66). Québec : Conseil québécois de la recherche sociale.

L'Écuyer, R. (dir.) (1990). Méthodologie de l'analyse développementale de contenu : méthode GPS et concept de soi. Québec : Presses de l'Université du Québec.

Lester, D. et Walker, R. L. (2007). Hopelessness, helplessness, and haplessness as predictors of suicidal ideation. Journal of Death and Dying, 55(4), 321-324. doi: 10.2190/OM.55.4.f

Lo, C. S., Ho, S. M. Y. et Hollon, S. D. (2010). The effects of rumination and depressive symptoms on the prediction of negative attributional style among college students. Cognitive Therapy and Research, 34(2), 116-123. doi: 10.1007/s10608-009-9233-2

Logan, T. K., Walker, R., Jordan, C. E. et Leukefeld, C. G. (2006). Internal contextual factors. Dans T. K. Logan, R. Walker, C. E. Jordan et C. G. Leukefeld (dir.), Women and victimization: Contributing factors, interventions, and implications (pp. 93-131). Washington, DC : American Psychological Association.

Lyon, M. E., Benoit, M., O’Donnell, R. M., Getson, P. R., Silber, T. et Walsh, T. (2000). Assessing African American adolescents' risk for suicide attempts: Attachment theory. Adolescence, 35(137), 121-134.

McGee, R. A., Wolfe, D. A. et Olson, J. (2001). Multiple maltreatment, attribution of blame, and adjustment among adolescents. Development and Psychopathology, 13, 827-846.

Mertens, D. M. (2005). Qualitative methods. Dans D.M. Mertens (dir.), Research and evaluation in education and psychology (2 éd.) (pp. 229-264). Thousand Oaks, CA : Sage.

Mezulis, A., Funasaki, K. et Hyde, J. S. (2011). Negative cognitive style trajectories in the transition to adolescence. Journal of Clinical Child and Adolescent Psychology, 40(2), 318-331. doi: 10.1080/15374416.2011.546048

Miles, M. B. et Huberman, A. M. (2003). Analyse des données qualitatives. Paris, France : De Boeck.

Ministère de la Santé et des Services sociaux du Québec. (2004). Lexiques. Consulté le 30 septembre 2011 à : http:// wpp01.msss.gouv.qc.ca/appl/M02/M02Lexique.asp

Morrow, K. B. (1991). Attributions of female adolescent incest victims regarding their molestation. Child Abuse \& Neglect, 15(4), 477-483. doi: 10.1016/0145-2134(91)90031-8

Nilsen, W. et Conner, K. R. (2002). The association between suicidal ideation and childhood and adult victimization. Journal of Child Sexual Abuse, 11(3), 49-62. doi : 10.1300/J070v11n03_03

O’Neill, M. L. et Kerig, P. K. (2000). Attributions of self-blame and perceived control as moderators of adjustment in battered women. Journal of Interpersonal Violence, 15(10), 1036-1049. doi: 10.1177/088626000015010002

Organisation mondiale de la santé. (2004). Le suicide. Dans Organisation mondiale de la santé (dir.), Rapport violences et santé (pp.102-124). Paris, France : Haut comité de la santé publique. 
Paillé, P. et Mucchielli, A. (2003). L'analyse qualitative en sciences humaines et sociales. Paris, France : Collin.

Putnam, F. W., Liss, M. B. et Landsverk, J. (1996). Ethical issues in maltreatment research with children and adolescents. Dans K. Hoagwood, P. S. Jensen et C. B. Fisher (dir.), Ethical issues in mental health research with children and adolescents (pp. 113-132). Hillsdale, NJ: Lawrence Erlbaum Associates.

Québec. (2007). Loi sur la protection de la jeunesse. L.R.Q., Chap. P-34.1.

Sneddon, H. (2003). The effects of maltreatment on children's health and well-being. Child Care in Practice, 9, 236250. doi: 10.1080/1357527032000167795

Steel, J., Sanna, L., Hammond, B., Whipple, J. et Cross, H. (2004). Psychological sequelae of childhood sexual abuse: Abuse-related characteristics, coping strategies, and attributional style. Child Abuse \& Neglect, 28(7), $785-801$. doi: 10.1016/j.chiabu.2003.12.004

Tourigny, M., Mayer, M., Wright, J., Lavergne, C., Trocmé, N., Hélie, S., . . Larrivée, M.-C. (2002). Étude sur l'incidence et les caractéristiques des situations d'abus, de négligence, d'abandon et de troubles de comportement sérieux signalés à la Direction de la protection de la jeunesse au Québec (ÉIQ). Montréal, QC : Centre de liaison sur l'intervention et la prévention psychosociales (CLIPP).

Valentine, L. et Feinauer, L. L. (1993). Resilience factors associated with female survivors of childhood sexual abuse. American Journal of Family Therapy, 21(3), 216-224. doi:10.1080/01926189308250920

Valle, L. A. et Silovsky, J. F. (2002). Attributions and adjustment following child sexual abuse and physical abuse. Child Maltreatment, 7(1), 9-25. doi: 10.1177/1077559502007001002

Webb, M., Heisler, D., Call, S., Chickering, S. A. et Colburn, T. A. (2007). Shame, guilt, symptoms of depression, and reported history of psychological maltreatment. Child Abuse \& Neglect, 31(11-12), 1143-1153. doi: 10.1016/j. chiabu.2007.09.003

Weiner, B. (1986). Attribution, emotion, and action. Dans R. M. Sorrentino et E. T. Higgins (dir.), Handbook of motivation and cognition : Foundations of social behavior (pp. 281-312). New York, NY : Guilford Press.

Zinzow, H., Seth, P., Jackson, J., Niehaus, A. et Fitzgerald, M. (2010). Abuse and parental characteristics, attributions of blame, and psychological adjustment in adult survivors of child sexual abuse. Journal of Child Sexual Abuse, 19, 79-98. doi: 10.1080/10538710903485989 